\title{
Bioanalysis
}

\section{HPLC 2015}

The HPLC 2015 Conference was held from the 21 to 25 June at the International Conference Center in Geneva, Switzerland. The emphasis of the meeting was around fundamental aspects of separations, sample preparation, novel developments and applications and hyphenation with MS. In this conference report, a selection of highlights of the Conference is given, based on the sessions attended by the authors, and focusing on subjects with possible relevance in the field of drug metabolism and bioanalysis. Selected papers from HPLC 2015 will be published in a virtual special issue of the Journal of Chromatography.

Keywords: biopharmaceuticals • ion mobility • metabolomics $\bullet$ multidimensional separations - supercritical fluid chromatography

\section{Overview}

HPLC 2015 was the 42nd Symposium of HPLC since its first edition in 1973. The meetings alternate between Europe and the USA with even years being in the USA. Since 2008 additional meetings are held in Asia and other continents. Over 1100 participants registered for the symposium. A conference dinner was held on Wednesday evening at a beautiful location at Coppet Castle, with great weather, nice food, good company, live music and a DJ!

The emphasis of the meeting was around fundamental aspects of separations, sample preparation, novel developments and applications and hyphenation with MS. With nine tutorials, 583 posters and 170 presentations in three parallel sessions, there was a lot to choose from. Also, it was impossible for us to attend all lectures and to give a complete overview of this conference. Therefore, in this report our (biased) highlights of the conference are given, based on the sessions we attended, focusing on subjects with possible relevance in the field of drug metabolism and bioanalysis that we are working in.

\section{Past, present \& future of LC-MS}

Jack Henion (Quintiles, USA) kicked off the Monday sessions with a plenary lecture titled 'Past, present and future of LC-MS.' LC-MS started in 1958 with the direct liquid interface. An important milestone was the invention of pneumatically assisted electrospray in the late 1980s. Some 'snapshots' were given for the present and the future of LC-MS, such as the development of an automated online extraction membrane to be able to obtain dried plasma spots from whole blood, screening of foods for pesticides using liquid extraction surface analysis, bioanalysis of large molecules using LC-MS and personalized MS, where a small mass spectrometer can be placed at the physician's office to check if the patients are on the drug medication. Last but not least, MS in the operating room was discussed. The use of desorption electrospray ionization mass spectrometric imaging (DESI-MSI) may be valuable in the future for routine intraoperative assessment of the specimen margins during cancer surgery for which surgical margin assessment is of critical importance [1].
Ronald de Vries*,1 \& Valerie Koppen ${ }^{1}$

'Janssen Research \& Development, Beerse, Belgium

*Author for correspondence:

Tel.: +32 14607366

rdvries@its.jnj.com
FUTURE
SCIENCE 


\begin{abstract}
Ion mobility
With ion mobility, gas-phase separation is obtained based on shape (collisional cross-section; CCS) and charge. Therefore, ion mobility is complementary to liquid chromatography and MS. Incorporating ion mobility into an LC-MS method has the potential to enhance the separation of analyte ions (e.g., in case of isobaric compounds) and to reduce chemical background noise. In two talks, examples were given in the area of both small molecule analysis using FAIMS ('Combining asymmetric waveform Ion Mobility Spectrometry with LC-MS for the analysis of small molecules and metabolites,' a lecture by Creaser Colin [Centre of Analytical Science, UK]) and tryptic peptide analysis using differential ion mobility separation (DMS, 'Sensitivity and selectivity evaluation of peptides quantification using ion mobility spectrometry and cubed selected reaction monitoring,' a lecture by Bandar Alghanem [University of Geneva]). In the latter case, the effect of organic modifiers in the transport gas was investigated and resulted in an enhanced peptide mobility separation and an additional dimension to tune the selectivity on the assay. Good results were obtained with isopropanol as modifier.
\end{abstract}

\section{Multidimensional separations}

There were many presentations on multidimensional separations. Andre de Villiers (Stellenbosch University) presented on the use of comprehensive HILIC $\times$ RP liquid chromatography. The method was optimized to avoid the need for flow splitting and in this way obtain maximum sensitivity. For HILIC in the first dimension, a $150 \times 1 \mathrm{~mm}$ column was used at a flow rate of $1 \mu \mathrm{l} / \mathrm{min}$ using a $315 \mathrm{~min}$ gradient. For RP separation in the second dimension, a $50 \times 2.1 \mathrm{~mm}$ column was used at a flow rate of $0.86 \mathrm{ml} / \mathrm{min}$ with a cycle time of 2 min. Passive modulation using a dual injection loop was used for the transfer of the fractions from the first to the second dimension.

Andrea Gargano (TI Coast, University of Amsterdam, The Netherlands) presented on 'High efficiency separations of peptides and proteins using actively modulated LC $\times$ LC-HRMS' [2]. In most LC $\times$ LC set ups, the first dimension column is typically narrow and long and the second dimension column is wide and short. Passive modulation is usually used in this approach, where the eluent from the first dimension is transferred to the second dimension via an injection loop. Limitations of this approach include low sample capacity in first dimension, a high dilution of the sample in the complete system and difficulties for splitless hyphenation with (nano) electrospray MS. With active modulation, the eluent from the first dimension is transferred to the second dimension via a trapping column. A make-up flow is used between first dimension column and trapping column to reduce the solvent strength. When active modulation is used, it is possible to use a larger column diameter in the first dimension than in the second dimension. As proof of principle, SCX $\times$ RP analysis was applied, using a first dimension $150 \times 0.3 \mathrm{~mm}$ column at $1 \mu \mathrm{l} / \mathrm{min}$. A make-up flow of $0.5 \mu \mathrm{l} / \mathrm{min}$ was applied. Two C18 $20 \times 0.3 \mathrm{~mm}$ trap columns were used for the active modulation, and a $50 \times 0.075 \mathrm{~mm}$ column was used in the second dimension.

An interesting approach for the separation of compounds in a wide polarity range was presented by Thomas Letzel (TU Munchen, Munich, Germany) in his lecture 'RPLC-HILIC versus SFC - Novel Screening techniques with Strongly Extended Polarity.' Serial coupling of RP and zwitterionic HILIC LC was applied. A $50 \times 3 \mathrm{~mm} \mathrm{RP}$ column was used at a flow-rate of $0.05 \mathrm{ml} / \mathrm{min}$. The low flowrate was used to be able to obtain a high percentage of organic modifier at the subsequent HILIC column. The RP column was connected to a $150 \times 2.1 \mathrm{~mm}$ ZIC-HILIC column through a T-piece. A binary pump delivered a gradient to the HILIC column at a flowrate of $0.4 \mathrm{ml} / \mathrm{min}$ through the T-piece. Both the RPLC gradient and the HILIC gradient were programmed in a smart way, so that in the first part of the run the more hydrophobic analytes were trapped on the RPLC column while the hydrophilic analytes were not retained by the RPLC column. The hydrophilic analytes were subsequently retained and separated by the HILIC column. In the second part of the run, the HILIC gradient was set at $40 \%$ aqueous. Now, the more hydrophobic analytes were separated on the RPLC column and not further retained by the HILIC column. This approach enables the separation of analytes with a wide variety of polarities [3].

\section{Supercritical fluid chromatography}

Quite some presentations as well as a tutorial were focused on supercritical fluid chromatography (SFC). The tutorial was titled 'Modern SFC - What the analytical chemists should know about it,' and was presented by Abhijit Tarafdera (Waters, USA). A short summary of the tutorial is given below.

SFC uses carbon dioxide as a solvent in chromatography. Because carbon dioxide is miscible with all the solvents used in normal phase and reversed phase HPLC and because of the compatibility with all the HPLC columns that are used, the SFC selectivity space is broader, encompassing normal and reversed phase HPLC selectivity space but also additional selectivity space [4]. SFC can provide orthogonality to HPLC selectivity which can help in identification of impuri- 
ties or degradants and in separation of analytes from matrix interferences. SFC can even be orthogonal to itself because of the wide range of columns and solvents that can be used. SFC is powerful in the separation of compounds with structural similarity, such as chiral compounds or positional isomers. The low viscosity of carbon dioxide results in higher diffusivity of analytes, resulting in a flatter van Deemter curve. As a consequence, the same separation efficiency can be obtained with SFC as for HPLC, but in a much faster analysis time. This is especially the case when using sub-2- $\mu \mathrm{m}$ particles, known as ultrahigh performance supercritical fluid chromatography or UHPSFC. The application space for SFC is broad, ranging from log Ps from -2 to 10 . A potential area for research is exploration of the use of SFC for more hydrophilic compounds, as the polarity limits where SFC can be used have not been fully explored yet. SFC can be hyphenated with MS through the use of an active backpressure regulator.

Jean-Luc Veuthey (University of Geneva) presented on 'New insights in SFC and SFC-MS for the analysis of drug substances.' As positive points for SFC and UHPSFC were mentioned the alternative selectivity compared with RPLC, the good retention of polar compounds, the possibility to couple UHPSFC with MS, the transfer to preparative scale (faster evaporation of mobile phase), high throughput chiral and achiral separation on one unique system, green technology (low consumption of organic solvents) and robustness and sensitivity being equivalent to UHPLC. Also negative points for SFC were mentioned. The choice of the dissolution solvent is more critical, coupling with MS, although possible, is less straightforward then with HPLC, and density gradients could be problematic to transfer from sub-2 $\mu \mathrm{m}$ to preparative scale. Also, the instrument is $20-30 \%$ more expensive than UHPLC, there is less possibility to tune the mobile phase, so different stationary phases are needed to tune chromatography, and $2.1 \mathrm{~mm}$ ID columns are hardly compatible with UHPSFC.

Caroline West (University of Orleans) presented on 'Development of an achiral SFC-MS screening strategy for impurity profiling of drug candidates.' On a selection of 160 drug candidates, different parameters were varied, such as the nature and concentration of the additive in the mobile phase and the stationary phase (23 different stationary phases were tested). An interesting and 'easy-to-use' chemometric approach was used for the optimization, making use of a Derringer desirability function [5].

\section{Metabolomics}

Thomas Hankemeier (Leiden University) presented on 'Remaining challenges in metabolomics, and strat- egies to address them.' Many metabolomics methods have been reported and various explorative biomarkers have been found. However, there is still a lack of standardization and mechanistic and clinical validation of biomarkers is required for clinical implementation. Currently, only a few validated metabolomics-based biomarkers are available. It is a challenge to find an appropriate study design which can properly capture the phenotype, which is often 'dynamic' [6]. Validation of results can also be a challenge, as data are often collected in different studies that need to be combined. An example was given of fusion of data of three different cohorts [7]. It was shown that LC-MS-based metabolomics platforms are robust and can handle many samples, but robust quantification is required to integrate different metabolomics studies. The future of metabolomics could further evolve from system diagnosis to integrated intervention and ultimately to personalized health.

\section{Biopharmaceuticals}

Christian Huber (University of Salzburg, Salzburg, Austria) presented on 'A top to bottom approach for in-depth characterization of therapeutic monoclonal antibodies.' The characterization was done at three different levels (top-down, middle-down and bottomup). Analysis at the intact protein level (top-down) confirms the overall molecular mass, provides information on the glycosylation pattern and reveals misassembled antibody fragments. A large influence of temperature on the separation was observed, which is probably caused by different conformations of the antibody. When the temperature was raised, one peak was observed. Analysis at light/heavy chain level (middledown) was done after either in-line electrochemical reduction or after FabRICATOR digestion to yield light and heavy chains. Analysis at this level confirms $\mathrm{C}$ - and N-terminal sequences and provides further information on the glycosylation pattern. Finally, analysis at the proteolytic peptide level (bottom-up) after tryptic digestion confirms the correct primary sequence and reveals posttranslational and artificial modifications including mutations, oxidation and deamidation. It was shown that analysis at all three structural levels can be realized on a single instrumental HPLC-MS platform. Suitable software is essential to interpret the large amount of data generated.

Nico van de Merbel (PRA Health Sciences) presented on 'Quantitative bioanalysis by LC-MS/MS: from small to large molecules. In this talk, different approaches for protein bioanalysis using LC-MS were discussed. Examples were shown where a signature peptide of a (pellet) digested protein was analyzed without further sample clean-up. Because of chro- 
matographic interferences, quantification limits are typically in the low $\mathrm{nM}$ range when this approach is used. Also, examples were shown where more selective sample preparation was used at the protein level, using immobilized metal affinity chromatography or at the peptide level using solid phase extraction. When protein and peptide extractions were combined, using immobilized metal affinity chromatography at the protein level and, after digestion, solid phase extraction for the signature peptide, sensitivity was further increased down to the low pM level.

\section{Conclusion}

HPLC2015 was an interesting and inspiring conference. The conference was very useful as a networking opportunity. Because of the variety of subjects, the conference was also useful to catch up and learn about

\section{References}

1 Eberlin LS, Tibshirani RJ, Zhang J et al. Molecular assessment of surgical-resection margins of gastric cancer by mass-spectrometric imaging. Proc. Natl Acad. Sci. USA 111(7), 2436-2441 (2014).

2 Vonk RJ, Gargano AFG, Davydova E et al. Comprehensive two-dimensional liquid chromatography with stationaryphase-assisted modulation coupled to high-resolution mass spectrometry applied to proteome analysis of Saccharomyces cervisiae. Anal. Chem. 87(10), 5387-5394 (2015).

3 Rajab M, Greco G, Heim C, Helmreich B, Letzel T. Serial coupling of RP and zwitterionic hydrophilic interaction LCMS: suspects screening of diclofenac transformation products by oxidation with a boron-doped diamond electrode. J. Sep Sci. 36(18), 3011-3018 (2013). subjects not directly related to daily work. It was a bit of a surprise that the far majority of oral presentations were from University and only a very limited number of presentations came from industry. Next congresses will be HPLC 2015 Asia in Beijing and HPLC 2016 in San Francisco.

\section{Financial \& competing interests disclosure}

The authors are employed at Janssen Research and Development and, therefore, receive compensation in the form of salary, stock and/or stock options. The authors have no other relevant affiliations or financial involvement with any organization or entity with a financial interest in or financial conflict with the subject matter or materials discussed in the manuscript apart from those disclosed.

No writing assistance was utilized in the production of this manuscript.

4 West C, Lesellier E. A unified classification of stationary phases for packed column supercritical fluid chromatography. J. Chromatog A 1191, 21-39 (2008).

5 Lemasson E, Bertin S, Hennig P et al. Development of an achiral supercritical fluit chromatography method with ultraviolet absorbance and mass s pectrometric detection for impurity profiling of drug candidates. Part 1: optimization of mobile phase composition. J. Chromatog A 1408, 217-226 (2015).

6 Ramautar R, Berger R, van der Greef J, Hankemeier T. Human metabolomics: strategies to understand biology. Curr. Opin. Chem. Biol. 17, 841-846 (2013).

7 Dane AD, Hendriks MMWB, Reijmers TH et al. Integrating metabolomics profiling measurements across multiple biobanks. Anal. Chem. 86, 4110-4114 (2014). 